\title{
Robust Delegation with Uncertain Monetary Policy Preferences
}

Marco M. Sorge

ISSN: 2031-4892

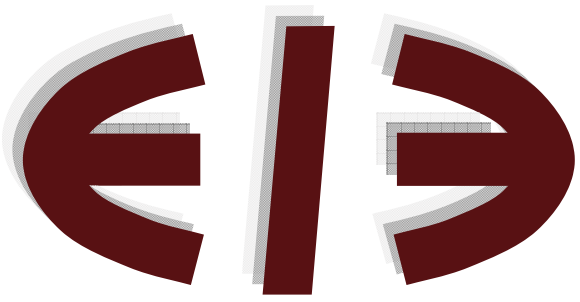

EERI

Economics and Econometrics Research Institute Avenue de Beaulieu 1160 Brussels

Belgium

Tel: +3222988491

Fax: +322 2988490

www.eeri.eu 


\title{
Robust Delegation with Uncertain Monetary Policy Preferences
}

\author{
Marco M. Sorge *
}

\begin{abstract}
Recent research has renewed interest in the exploration of the optimal design of monetary policy institutions in the presence of uncertainty. In this paper, we revisit the rationale for delegation to a weight-conservative central banker when the social planner's knowledge about the true preferences of delegates is ex ante ambiguous and he exhibits a preference for robustness. In this context, a robust (worst-case oriented) delegation strategy is intended to minimize the maximum welfare loss over the uncertainty set, when no prior probability distribution for the preference bias (conservatism-gap) is available. We find that both over and underconservatism may emerge with respect to the certainty case, for robust delegation is shown to be model-dependent. Most importantly, under reasonable model's parameterizations, Rogoff's principle is reversed: it is optimal for society to appoint a weight-liberal central banker.
\end{abstract}

Keywords: Delegation; Conservative central bank; Preference uncertainty; Minmax policy JEL Classification:E520; E580

*DISES - University of Salerno, Via Ponte don Melillo, 84084 Fisciano (SA), Italy - E-mail: marco.m.sorge@gmail.com 


\section{Introduction}

In recent years, several studies have emphasized the importance of model uncertainty for the conduct and performance of monetary policy (e.g. Levin and Williams, 2003; Onatsky and Williams, 2003; Leitemo and Soderstrom, 2008; Dennis, 2010). As a natural step forward, this strand of research has renewed interest in the exploration of the optimal design of monetary policy institutions under several sources of model misspecification and uncertainty (e.g. Beetsma and Jensen, 1998; Muscatelli, 1998; Tillmann, 2009a; Hefeker and Zimmer, 2011).

The present paper aims at contributing to this literature by addressing the case of robust monetary policy delegation in the presence of ex ante ambiguity about the true preferences of delegates, once in office ${ }^{1}$. Preference uncertainty is commonly presumed to exhibit a crucial influence on the regulation of monetary policy (e.g. Cukierman and Meltzer, 1986; Vickers, 1986; Blinder et al., 2008), and hence should be taken into account within the delegation process ${ }^{2}$. In this respect, Tillmann (2008) studies the welfare costs to society of appointing a central bank who exhibits a conservatism-gap with respect to the optimal scheme according to Rogoff (1985). While Tillmann (2008) only allows for an ex post deviation in the central banker's degree of conservatism, we rather explore the problem from an ex ante perspective, i.e. we investigate the optimal delegation scheme in the presence of imperfect predictability of monetary policy due to uncertain central bank preferences.

Specifically, we revisit Rogoff's (1985) legislative approach to delegation of monetary policy when the social planner's knowledge about the true degree of conservatism of delegates is ex ante ambiguous and he has a preference for robustness ${ }^{3}$. The notion of Knightian uncertainty is employed to capture situations where randomness surrounding a decision-making problem can not be described by a probability measure. We identify the delegation incentives faced by an ambiguity averse social planner by assuming that the latter is unable to assign any prior probability distribution to the monetary policy preferences bias. In order to hedge against this form of uncertainty, he then adopts

\footnotetext{
${ }^{1}$ Tillmann (2009b) is the first to introduce the notion of robust delegation. Nonetheless, he analyzes the mechanism of robust delegation when the social planner faces uncertainty about the persistence of cost-push shocks. Diana and Sidiropoulos (2007) and Tillmann (2009a) both address the problem of how to design an optimal delegation arrangement in the sense of Rogoff (1985), when the policy-maker faces unstructured (Knightian) model uncertainty.

${ }^{2}$ In fact, Rogoff's (1985) delegation approach raises the issue of the identification of the parameter governing the central banker's preferences with respect to conflicting objectives.

${ }^{3}$ In this regard, our paper differs from Qin et al. (2010), who study robust monetary policy when society is unaware of the true preferences for robustness of the policy-maker.
} 
a minmax approach (e.g. Hansen and Sargent, 2008) to delegation. A robust (worstcase oriented) delegation mechanism is intended to minimize the expected welfare loss to society over the uncertainty set. Following Tillmann (2009b), we refer to such an optimal delegation arrangement as robust delegation.

We prove that society's incentives to delegation of monetary policy responsibility to an independent central bank are dramatically altered by the presence of preference uncertainty, as conjectured in Tillmann (2008). Specifically, we show that both over and underconservatism may emerge with respect to the certainty case, contingent on the configuration of the worst-case scenario, which is model-dependent. While there exist cases in which no robust delegation arrangement exists, under reasonable parameterizations of the model and a sufficiently high degree of uncertainty, Rogoff's principle is reversed: society's optimal commitment towards fighting inflation via monetary policy delegation results in the appointment of a more liberal central banker than society's ${ }^{4}$.

The analysis presented here is related to different lines of research. Previous studies on the role of asymmetric information in monetary policy-making, in which the public is uncertain about the central banker's type (among others, Backus and Driffill, 1985; Cukierman and Meltzer, 1986; Vickers, 1986; Tabellini, 1988), differ from the present one in that they typically focus on the problem of preferences inference on the part of agents endowed with limited information, and how the central bank's decision to preserve its reputation may help to overcome the stabilization bias arising under discretionary monetary policy. Rather, our goal is to evaluate the optimal appointment scheme in the sense of Rogoff (1985), when the social planner is ambiguity averse. Remarkably, in our setting the monetary policy stage remains a complete information game as in Rogoff (1985), since upon conclusion of the delegation process, the central banker's preferences are assumed to be fully observable by the private agents ${ }^{5}$.

More closely related to our analysis are the seminal contributions of Beetsma and Jensen (1998) and Muscatelli (1998), who investigate the role of uncertain central bank preferences for the optimal design of monetary institutions. A central implication of these studies is that a higher degree of conservatism may be advisable for it constrains the volatility of monetary policy behavior in the presence of uncertainty. Our analysis differs from this work along two relevant dimensions. First, from a modelling perspective, the

\footnotetext{
${ }^{4}$ Alternative arguments for this result have been offered, among others, in the works of Guzzo and Velasco (1999), Lagerlöf (2001) and Lippi (2002).

${ }^{5}$ As a consequence, the monetary policy outcome is the usual inflationary equilibrium. The implications of incomplete information on the central banker's type for the optimal design of monetary policy institutions have been discussed in Sibert (2002) with respect to the delegation approach. See also D'Amato (2004).
} 
uncertainty introduced by the preference bias in our model is Knightian (ambiguity), while Beetsma and Jensen (1998) and Muscatelli (1998) deal with several forms of stochastic uncertainty. Second, our model's predictions are only in part in line with those derived in the mentioned papers, for we show that preference uncertainty may well drive the delegation choice towards a less conservative or even weight-liberal central banker.

The paper proceeds as follows. Section 2 briefly reviews standard results about optimal policy delegation under certainty, which are then used as a benchmark. In Section 3 we introduce Knightian uncertainty about monetary policy preferences and discuss the case of robust delegation. Section 4 offers concluding remarks.

\section{Optimal policy delegation under certainty}

We consider the canonical version of the New-Keynesian sticky-price general equilibrium model (e.g. Walsh, 2003), where inflation dynamics are captured by a forward-looking Phillips curve (1). Here $\pi_{t}$ is the inflation rate, $x_{t}$ the output gap, and $E_{t}$ denotes conditional expectations:

$$
\pi_{t}=\beta E_{t}\left(\pi_{t+1}\right)+\kappa x_{t}+e_{t}, \quad \beta \in(0,1), \quad \kappa>0
$$

Let the state of motion of $e_{t}$ be described by the following $\operatorname{AR}(1)$ process:

$$
e_{t}=\rho e_{t-1}+\epsilon_{t}, \quad \epsilon_{t} \sim N(0,1)
$$

with $\rho \in(0,1)$. Hence, the cost-push shock exhibit a nonzero degree of persistence.

Monetary policy is intended to minimize the loss function $\mathcal{L}$, which is in the form of a weighted sum of inflation volatility and output gap volatility ${ }^{6}$ :

$$
\mathcal{L}:=E_{0}\left\{\sum_{t=0}^{\infty} \beta^{t}\left(\pi_{t}^{2}+\lambda x_{t}^{2}\right)\right\}, \quad \lambda>0
$$

Under discretionary policy, optimal decision-making does not involve commitment to any future actions. Hence, the monetary authority takes expectations as given and selects

\footnotetext{
${ }^{6}$ More precisely, it can be shown that the welfare losses experienced by the representative household are proportional to (3) up to a second order approximation (e.g. Walsh, 2003). Without loss of generality, the target values for output and inflation are normalized to zero. The sequence of constraints (1) are sufficient to determine the equilibrium path for output and inflation under the optimal policy.
} 
$\left(x_{t}, \pi_{t}\right)$ in order to solve:

$$
\min _{x_{t}, \pi_{t}}\left\{\pi_{t}^{2}+\lambda x_{t}^{2}\right\}
$$

subject to (1). The optimality conditions for the problem above imply:

$$
\kappa \pi_{t}+\lambda x_{t}=0, \quad t=0,1, \ldots
$$

from which equilibrium inflation and output gap result in the following:

$$
\begin{aligned}
\pi_{t, d i s} & =\frac{\lambda}{\lambda(1-\beta \rho)+\kappa^{2}} e_{t} \\
x_{t, d i s} & =\frac{-\kappa}{\lambda(1-\beta \rho)+\kappa^{2}} e_{t}
\end{aligned}
$$

It is well-known that, in the presence of serially correlated cost-push shocks, discretionary policy suffers from a stabilization bias, i.e. inflation proves inefficiently stabilized with respect to the commitment case $^{7}$. The stabilization bias problem can be removed through delegation to a weight-conservative central banker, i.e when the social planner delegates responsibility for monetary policy to an independent central bank which places a relatively lower weight $\lambda_{C B}$ on the output gap objective. More specifically, for a given $\lambda_{P}$, with which the social planner weights fluctuations in the output gap, the (socially) optimal degree of conservatism $\lambda_{C B}$ is characterized as the solution of:

$$
\min _{\lambda_{C B}}\left\{\left\{\frac{\lambda_{C B}}{\lambda_{C B}(1-\beta \rho)+\kappa^{2}}\right\}^{2}+\lambda_{P}\left\{\frac{-\kappa}{\lambda_{C B}(1-\beta \rho)+\kappa^{2}}\right\}^{2}\right\}
$$

which yields:

$$
\lambda_{C B}=\lambda_{P}(1-\beta \rho)
$$

Since $\beta \rho<1$, the optimal weight attached to output gap fluctuations under certainty (i.e. the degree of central banker's conservatism) is lower than society's. The central bank is weight-conservative in the sense of Rogoff (1985).

\footnotetext{
${ }^{7}$ That is, $\operatorname{var}\left(\pi_{t, \text { dis }}\right)>\operatorname{var}\left(\pi_{t, \text { rule }}\right)$, where $\pi_{t, \text { rule }}$ is the equilibrium inflation resulting under commitment and $\operatorname{var}(\cdot)$ denotes unconditional variance.
} 


\section{Robust delegation under preference uncertainty}

Let now the social planner be uncertain about the true preferences of the appointed central banker, once in office. Specifically, for any selected $\lambda_{C B}$, the policy-maker will exhibit a conservatism-gap $\delta$ and hence place the weight $\lambda_{C B}(1+\delta)$ on output gap stabilization relative to his inflation objective (Tillmann, 2008). A crucial assumption here is that the social planner is unable to assign any probability distribution to the monetary policy preference bias $\delta$, which is only known to reside in a bounded interval:

$$
|\delta| \leq \bar{\delta}, \quad 0<\bar{\delta}<1
$$

We interpret $\bar{\delta}$ as a measure of the ex ante preference uncertainty present in the economy, which is assumed to fully resolve once the delegation stage has occurred ${ }^{8}$.

Apparently, given that both the optimal (discretionary) policy and the resulting stabilization bias - hence, the delegation choice - depend on the actual monetary policy preferences with respect to conflicting objectives, preference uncertainty represents an important force governing the decision to delegate monetary policy responsibility in the sense of Rogoff (1985). Since no prior over the interval of possible realizations of $\delta$ exists, the social planner will adopt a minmax approach to delegation such that the worst possible welfare loss due to conservatism bias is minimized. This worst-case scenario constitutes the outcome against which the social planner wants the delegation scheme, captured by the controllable instrument $\lambda_{C B}$, to be robust.

Technically, the robust counterpart to (8) is:

$$
\min _{\lambda_{C B}}\left\{\max _{|\delta| \leq \bar{\delta}} E\left\{\mathcal{L}_{t}\left(\pi_{t, d i s}\left(\lambda_{C B}, \delta\right), x_{t, d i s}\left(\lambda_{C B}, \delta\right)\right)\right\}\right\}
$$

where $\mathcal{L}_{t}:=\pi_{t, d i s}^{2}+\lambda_{P} x_{t, d i s}^{2}$.

The Problem (11) possesses a nontrivial solution, which is defined in terms of nonlinear constraints expressing the dependence of the inner (maximization) problem's solution on the model's parameters. This in turn involves jump discontinuities in the (piecewise continuous) maximum value function. The following Proposition characterizes the robust delegation arrangement in the presence of uncertain monetary policy preferences:

\footnotetext{
${ }^{8}$ As a consequence, in the monetary policy stage the central banker's type $\lambda_{C B}(1+\delta)$ is common knowledge. This implies that the incentive to exploit the central banker's degree of conservativeness as a commitment device is not driven by nonstandard strategic interactions between the policymaker and the private agents.
} 
Proposition 1. The optimal (robust) degree of monetary policy conservatism under uncertainty is model-dependent. In particular, for given $\left(\lambda_{P}, \beta, \rho, \kappa\right)$ :

i) If $\lambda_{P}(1-\beta \rho)^{2}=\kappa^{2}$, then $\lambda_{C B} \in\left\{\Gamma_{0}, \Gamma_{1}\right\}$ where:

$$
\Gamma_{0}:=\frac{\lambda_{P}(1-\beta \rho)}{1+\bar{\delta}}, \quad \Gamma_{1}:=\frac{\lambda_{P}(1-\beta \rho)}{1-\bar{\delta}}
$$

ii) If $\bar{\delta} \kappa^{2}<\lambda_{P}(1-\beta \rho)^{2}<\kappa^{2}$, then no solution to (11) exists;

iii) If $\lambda_{P}(1-\beta \rho)^{2}>\kappa^{2}$ or $\lambda_{P}(1-\beta \rho)^{2} \leq \bar{\delta} \kappa^{2}$, then $\lambda_{C B}=\Phi$, where:

$$
\Phi:=\frac{\lambda_{P}(1-\beta \rho)^{2}-\kappa^{2}+\sqrt{\lambda_{P}^{2}(1-\beta \rho)^{4}+\kappa^{4}+2 \lambda_{P}(1-\beta \rho)^{2} \kappa^{2}\left(1-2 \bar{\delta}^{2}\right)}}{2(1-\beta \rho)\left(1-\bar{\delta}^{2}\right)}
$$

Proof. - See the Appendix.

Under robust delegation, the optimal degree of central bank's conservatism is always attuned according to the degree of preference uncertainty $\delta$, which is either underestimated $(\delta=-\bar{\delta})$ or overestimated $(\delta=\bar{\delta})$ within the optimal delegation scheme. Since $\Gamma_{0}<$ $\lambda_{P}(1-\beta \rho)<\Gamma_{1}$, under the knife-edge case i), robust delegation may either involve underconservatism or overconservatism with respect to the certainty scenario, as these both lead to the same expected maximum welfare loss. Also, whenever $\beta \rho<\bar{\delta}^{9}$, it could be socially optimal to appoint a weight-liberal central banker, i.e. one who cares more about output stabilization and less about inflation, relative to what would be the case in the standard Rogoff's environment. This occurs as the presence of uncertainty renders delegation more costly, and may weaken the need for conservatism. In fact, the expected welfare cost from distortion in stabilization policy is in principle able to outweigh the expected gain from lower inflation. Under particular circumstances, the effect of uncertainty - loss from wrong appointment - counteracts the familiar Rogoff's effect - removal of the stabilization bias, if the latter is not too severe. As a consequence, delegating the conduct of monetary policy to a weight-conservative central banker is no longer optimal. The same occurs under case iii $)^{10}$, if it holds that $\lambda_{P}(1-\beta \rho)\left(2 \bar{\delta}^{2}-1-\beta \rho\right)>$ $\kappa^{2}$. Finally, under case ii), the solution set of the robust optimization problem underlying the delegation decision is empty.

To evaluate the effects of preference uncertainty on the delegation arrangement, we

\footnotetext{
${ }^{9}$ This restriction is more likely to occur the lower the (discounted) persistence of cost-push shock and the higher the degree of preference uncertainty present in the economy. As an example, for the policy scenario presented in Table 1 , this inequality would hold for any $\bar{\delta} \in(0.297,1)$.

${ }^{10}$ More generally, since $\Phi>\lambda_{P}(1-\beta \rho)$, case iii) is always characterized by underconservatism with respect to the certainty scenario.
} 
introduce a standard parameterization of the basic New-Keynesian model, which is reported in Table $1^{11}$. We set the degree of preference uncertainty to $\bar{\delta}=0.9$. According to Proposition 1, the robust delegation arrangement involves setting $\lambda_{C B}=\Phi$, with $\Phi>\lambda_{P}$. It follows that, in contrast with Rogoff's analysis, the optimally chosen central banker is more weight-liberal than society's itself ${ }^{12}$.

Table 1. Robust delegation under standard parameterization

\begin{tabular}{cccccc}
\hline \hline$\beta$ & $\rho$ & $\kappa$ & $\lambda_{P}$ & $\bar{\delta}$ & $\Phi\left(=\lambda_{C B}\right)$ \\
\hline 0.99 & 0.30 & 0.05 & 0.25 & 0.9 & $\approx 0.909$ \\
\hline
\end{tabular}

We emphasize that the particular (multiplicative) structure of uncertainty considered in our model is not pivotal for the results. Importantly, analogous findings would emerge if additive uncertainty, as in Beetsma and Jensen (1998), were to be considered instead. Also, the same would hold true if the social planner were to follow a less preserving (relative) robustness strategy, which would be rather designed to minimize the maximum regret associated with failing to select the optimal central banker's type for any given realization of the uncertain input $\delta$. Specifically, a relative robust solution is the outcome of the following problem:

$$
\min _{\lambda_{C B}}\left\{\max _{|\delta| \leq \bar{\delta}} E\left\{\mathcal{L}_{t}-\mathcal{L}_{t}^{*}\right\}\right\}
$$

where $\mathcal{L}_{t}^{*}$ is the optimal value function for a given $\delta:|\delta| \leq \bar{\delta}$ (fixed), i.e.:

$$
\mathcal{L}_{t}^{*}:=\min _{\lambda_{C B}} \pi_{t, d i s}^{2}\left(\lambda_{C B}(\delta), \delta\right)+\lambda_{P} x_{t, d i s}^{2}\left(\lambda_{C B}(\delta), \delta\right)
$$

In fact, we have the following:

Proposition 2. Problem (12) is equivalent to Problem (11), i.e. they possess the same solution set.

Proof. - See the Appendix.

\footnotetext{
${ }^{11}$ The employed parameter values comply with both the theoretical and the empirical literature on New-Keynesian models (e.g. Galí and Gertler, 1999).

${ }^{12}$ A notable implication of Proposition 1 concerns the response of the optimal output weight of the central bank when the economy faces larger uncertainty about monetary policy preferences. Since $\partial \Gamma_{0} / \partial \bar{\delta}<0, \partial \Gamma_{1} / \partial \bar{\delta}>0$ and $\partial \Phi / \partial \bar{\delta}>0$, the former may either increase or decrease with the degree of preference uncertainty $\bar{\delta}$, given model-specific parameter restrictions. This suggests that uncertainty may have asymmetric effects on the optimal design of the monetary policy institution.
} 


\section{Conclusion}

In this paper, we have revisited the rationale for delegation to a weight-conservative central banker in the presence of Knightian uncertainty about monetary policy preferences. In the standard New-Keynesian model of monetary policy delegation under preference uncertainty, we have shown that society's incentives to delegate to a weight-conservative central banker may be amplified, mitigated or even reversed, contingent on model-specific parameter restrictions. This finding might help to explain why the delegation of the conduct of monetary policy to a weight-conservative (independent) central banker is highly debated on both theoretical and practical levels. 


\section{Appendix}

\section{Proof of Proposition 1}

The minmax problem (11) can be recast as a two-level optimization program:

$$
\begin{array}{cc}
\min _{\lambda_{C B}} & f\left(\lambda_{C B}, \delta\left(\lambda_{C B}\right)\right) \\
\text { s.t. } & \\
\delta\left(\lambda_{C B}\right) \in \arg \max _{\delta} f\left(\lambda_{C B}, \delta\right) \\
\text { s.t. }|\delta| \leq \bar{\delta}
\end{array}
$$

where:

$$
f\left(\lambda_{C B}, \delta\right):=\left\{\frac{\lambda_{C B}^{2}(1+\delta)^{2}+\lambda_{P} \kappa^{2}}{\left[\lambda_{C B}(1+\delta)(1-\beta \rho)+\kappa^{2}\right]^{2}}\right\}
$$

The function $f$ is strictly quasiconvex in $\delta$ for fixed (nonzero) $\lambda_{C B}$, and is such that $\partial f / \partial \delta<0$ for $\delta<\delta^{*}$ and $\partial f / \partial \delta>0$ for $\delta>\delta^{*}$, with $\delta^{*}:=\lambda_{C B}^{-1} \lambda_{P}(1-\beta \rho)-1$. Hence, contingent on the value of $\lambda_{C B}$ and the model's parameters $\left(\lambda_{P}, \beta, \rho, \kappa\right)$, the function $f$ attains the maximum value at its boundary, i.e. either at $-\bar{\delta}$ or $\bar{\delta}$ (or both). For given $\left(\lambda_{P}, \beta, \rho, \kappa\right)$, define:

$$
\begin{gathered}
f_{1}:=f\left(\lambda_{C B}, \delta=-\bar{\delta}\right), \quad f_{2}:=f\left(\lambda_{C B}, \delta=\bar{\delta}\right) \\
\Omega:=\frac{\lambda_{P}(1-\beta \rho) \kappa^{2}}{\kappa^{2}-\lambda_{P}(1-\beta \rho)^{2}} \quad\left(\kappa^{2} \neq \lambda_{P}(1-\beta \rho)^{2}\right)
\end{gathered}
$$

We make use of the following ${ }^{13}$ :

Lemma 1. Let $\left(\lambda_{P}, \beta, \rho, \kappa\right)$ be fixed. Then:

i) $\Omega>\lambda_{P}(1-\beta \rho) \Leftrightarrow \kappa^{2}>\lambda_{P}(1-\beta \rho)^{2}$;

ii) $\Omega<\Gamma_{1} \quad \Leftrightarrow \quad \lambda_{P}(1-\beta \rho)^{2}>\bar{\delta} \kappa^{2}$;

iii) $\lambda_{P}(1-\beta \rho)<\Phi<\Gamma_{1}$;

iv) If $\kappa^{2}>\lambda_{P}(1-\beta \rho)^{2}$, then $\Phi<\Omega$;

v) $\exists$ ! $\tilde{\lambda}_{C B} \in(0, \infty): f_{1}\left(\tilde{\lambda}_{C B}\right)=f_{2}\left(\tilde{\lambda}_{C B}\right)$.

Lemma 2. Let $\left(\lambda_{P}, \beta, \rho, \kappa\right)$ be fixed. Then:

i) If $\lambda_{P}(1-\beta \rho)^{2}=\kappa^{2}$, then $\bar{\delta}=\arg \max _{\delta} f\left(\operatorname{resp} . \quad-\bar{\delta}=\arg \max _{\delta} f\right)$ if and only if

\footnotetext{
${ }^{13}$ Lemma 1 is trivial. The proof of Lemma 2 , which is based upon contrasting $f_{1}$ with $f_{2}$ for any given $\lambda_{C B}$, requires straightforward yet tedious calculations and is therefore omitted for ease of exposition. Full details are available upon request.
} 
$\kappa \sqrt{\lambda_{P}}<\lambda_{C B} \leq \kappa \sqrt{\frac{\lambda_{P}}{1-\delta^{2}}}$ (resp. $\lambda_{C B} \leq \kappa \sqrt{\lambda_{P}}$ or $\lambda_{C B} \geq \kappa \sqrt{\frac{\lambda_{P}}{1-\delta^{2}}}$ );

ii) If $\lambda_{C B}<\Gamma_{0}$ (resp. $\lambda_{C B}>\Gamma_{1}$ ), then $-\bar{\delta}=\arg \max _{\delta} f\left(\right.$ resp. $\left.\bar{\delta}=\arg \max _{\delta} f\right)$;

iii) If $\Gamma_{0} \leq \lambda_{C B} \leq \lambda_{P}(1-\beta \rho)$, then $-\bar{\delta}=\arg \max _{\delta} f$;

iv) If $\lambda_{P}(1-\beta \rho)<\lambda_{C B}<\Omega$, then $\bar{\delta}=\arg \max _{\delta} f$;

v) If $\max \{\Omega, \Phi\}<\lambda_{C B} \leq \Gamma_{1}$, then $\bar{\delta}=\arg \max _{\delta} f$

According to Lemma 2, contingent on the model's parameters ${ }^{14}$, the maximum value function $\max _{\delta} f\left(\lambda_{C B}, \delta\right)$ is defined as a piecewise (continuous) function:

Case 1: $\lambda_{P}(1-\beta \rho)^{2}=\kappa^{2}$

$$
\max _{\delta} f=\left\{\begin{array}{l}
f_{1} \quad \text { if } \lambda_{C B} \in\left[0, \kappa \sqrt{\lambda_{P}}\right] \cup[\Phi,+\infty) \\
f_{2} \text { if } \lambda_{C B} \in\left(\kappa \sqrt{\lambda_{P}}, \Phi\right]
\end{array}\right.
$$

Case 2: $\Gamma_{1}>\Omega>\lambda_{P}(1-\beta \rho)$

$$
\max _{\delta} f=\left\{\begin{array}{l}
f_{1} \quad \text { if } \lambda_{C B} \in\left[0, \lambda_{P}(1-\beta \rho)\right] \cup\{\Phi\} \\
f_{2} \quad \text { if } \lambda_{C B} \in\left(\lambda_{P}(1-\beta \rho), \infty\right)
\end{array}\right.
$$

Case 3: $\Omega<0$ or $\Omega \geq \Gamma_{1}$

$$
\max _{\delta} f=\left\{\begin{array}{l}
f_{1} \quad \text { if } \lambda_{C B} \in[0, \Phi] \\
f_{2} \text { if } \lambda_{C B} \in[\Phi,+\infty)
\end{array}\right.
$$

We now exploit the fact that $f_{1}$ (resp. $f_{2}$ ), defined on $\Re_{+}$as a function of $\lambda_{C B}$, possesses a strict global minimum at $\Gamma_{1}\left(\right.$ resp. $\left.\Gamma_{0}\right)$. In Case $1, \Gamma_{1}$ (resp. $\left.\Gamma_{0}\right)$ lies in the subinterval on which $\max _{\delta} f=f_{1}\left(\operatorname{resp} \cdot \max _{\delta} f=f_{2}\right)$. It therefore holds:

$$
f_{1}\left(\Gamma_{1}\right)=f_{2}\left(\Gamma_{0}\right) \quad \Rightarrow \quad \lambda_{C B} \in\left\{\frac{\lambda_{P}(1-\beta \rho)}{1+\bar{\delta}}, \frac{\lambda_{P}(1-\beta \rho)}{1+\bar{\delta}}\right\}
$$

In Case 2, $f_{1}$ (resp. $f_{2}$ ) is monotonically decreasing (resp. increasing) in the subintervals

\footnotetext{
${ }^{14}$ Specifically, what matters is the ordering of $\lambda_{P}(1-\beta \rho)$ and $\Omega$ on the real line with respect to the interval $\left[\Gamma_{0}, \Gamma_{1}\right]$.
} 
in which $\max _{\delta} f=f_{1}$ (resp. $\max _{\delta} f=f_{2}$ ). Hence, while $f_{1}$ is minimized at $\Phi$ (and $\left.f_{1}(\Phi)=f_{2}(\Phi)\right), f_{2}$ has an infimum at $\lambda_{P}\left(1-\beta \rho\right.$ ), where nonetheless $\max _{\delta} f=f_{1}$ (and $\left.f_{1}\left(\lambda_{P}(1-\beta \rho)\right)>f_{1}(\Phi)\right)$. As a consequence, no solution to the minmax problem (11) exists.

Finally, Case 3 is analogous to Case 2, with the exception that both $f_{1}$ and $f_{1}$ are minimized at $\Phi$. From $f_{1}(\Phi)=f_{2}(\Phi)$, it follows that $\lambda_{C B}=\Phi$.

\section{Proof of Proposition 2}

It is straightforward to note that, for a given $\delta:|\delta| \leq \bar{\delta}$ (fixed), we have:

$$
\lambda_{C B}^{\prime}=\arg \min _{\lambda_{C B}} \mathcal{L}_{t}\left(\lambda_{C B}, \delta\right)=\frac{\lambda_{P}(1-\beta \rho)}{1+\delta}
$$

and hence:

$$
\mathcal{L}_{t}^{*}:=\pi_{t, d i s}^{2}\left(\lambda_{C B}^{\prime}, \delta\right)+\lambda_{P} x_{t, d i s}^{2}\left(\lambda_{C B}^{\prime}, \delta\right)=\left\{\frac{\lambda_{P}}{\lambda_{P}(1-\beta \rho)^{2}+\kappa^{2}}\right\}
$$

Since $\mathcal{L}_{t}^{*}$ is independent of both $\lambda_{C B}$ and $\delta$, the solution set of (12) corresponds to the solution set of (11).

\section{References}

[1] Backus, D., Driffil, J., 1985. Inflation and reputation. American Economic Review $75,530-538$.

[2] Beetsma, R.M.W.J., Jensen, H., 1998. Inflation targets and contracts with uncertain central bank preferences. Journal of Money, Credit, and Banking 30, 384-403.

[3] Blinder, A., Ehrmann, M., Fratscher, M., de Haan, J., Jansen, D., 2008. Central bank communication and monetary policy: a survey of theory and evidence. Journal of Economic Literature 46, 910-945.

[4] Cukierman, A., Meltzer, A. H., 1986. A theory of ambiguity, credibility, and inflation under discretion and asymmetric Information. Econometrica 54, 1099-1128.

[5] D'Amato, M., 2004. Commitment of monetary policy with uncertain central bank preferences. CSEF Working Papers 11\%, Centre for Studies in Economics and Finance. 
[6] Dennis, R., 2010. How robustness can lower the cost of discretion. Journal of Monetary Economics 57, 653-667.

[7] Diana, G., Sidiropoulos, M., 2007. Robust control and monetary policy delegation, 303-310. In: Salzano, M., Colander, D. (eds.), Complexity hints for Economic Policy, Springer Milan.

[8] Galí, J., Gertler, M., 1999. Inflation dynamics: A structural econometric investigation. Journal of Monetary Economics 44, 195-222.

[9] Guzzo, V., Velasco, A., 1999. The case for a populist central Banker. European Economic Review 43, 1317-1344.

[10] Hansen, L.P., Sargent, T.J., 2008. Robustness. Princeton University Press, Princeton.

[11] Hefeker, C., Zimmer, B., 2011. Central bank independence and conservatism under uncertainty: Substitutes or complements?. CESifo Working Paper Series 3344 , CESifo Group Munich.

[12] Lagerlöf, J., 2001. Costly information acquisition and delegation to a "liberal" central banker. Economics \&f Politics 13, 221-236.

[13] Leitemo, J., Söderström, U., 2008. Robust monetary policy in the New Keynesian framework. Macroeconomic Dynamics 12, 126-135.

[14] Levin, A.T., Williams, J.C., 2003. Robust monetary policy with competing reference models. Journal of Monetary Economics 50, 945-975.

[15] Lippi, F., 2002. Revisiting the case for a populist central banker. European Economic Review 46, 601-612.

[16] Muscatelli, A., 1998. Optimal inflation contracts and inflation targets with uncertain central bank preferences: Accountability through independence?. Economic Journal $108,529-542$.

[17] Onatski, A., Williams, N., 2003. Modeling model uncertainty. Journal of the European Economic Association 1, 1087-1122.

[18] Qin, L., Sidiropoulos, M., Spyromitros, E., 2010. Robust monetary policy under uncertainty about central bank preferences. Bulletin of Economic Research 62, 197 208. 
[19] Rogoff, K., 1985. The optimal degree of commitment to an intermediate monetary target. Quarterly Journal of Economics 100, 1169-1189.

[20] Sibert, A., 2002. Monetary policy with uncertain central bank preferences. European Economic Review 46, 1093-1109.

[21] Tabellini, G., 1988. Centralized wage setting and monetary policy in a reputational equilibrium. Journal of Money, Credit and Banking 20, 102-118.

[22] Tillmann, P., 2008. The conservative central banker revisited: Too conservative is more costly than too liberal. European Journal of Political Economy 28, 941-741.

[23] Tillmann, P., 2009a. Does model uncertainty justify conservatism? Robustness and the delegation of monetary policy. The B.E. Journal of Macroeconomics (Contributions) 9, Article 26.

[24] Tillmann, P., 2009b. The stabilization bias and robust monetary policy delegation. Journal of Macroeconomics 31, 730-734.

[25] Vickers, J., 1986. Signalling in a model of monetary policy with incomplete information. Oxford Economic Papers 38, 443-455.

[26] Walsh, C., 1995. Optimal contracts for central bankers. American Economic Review 85, 150-167.

[27] Walsh, C., 2003. Monetary theory and Policy. MIT Press, Cambridge. 